Pacific Journal of Mathematics

COMMUTATIVITY AND IDEALS IN THE BIDUALS OF 


\title{
COMMUTATIVITY AND IDEALS IN THE BIDUALS OF TOPOLOGICAL ALGEBRAS
}

\author{
S. L. GULICK
}

Earlier there has been described on the bidual (second conjugate space) of each member of a large class of topological algebras an Arens multiplication which makes it again into a topological algebra and which extends the multiplication of the original algebra. This paper centers around three questions concerning this Arens multiplication.

In the first place, we characterize those commutative algebras whose biduals are also commutative. We then discuss the extent to which the passing of commutativity from the base algebra to the bidual is preserved under certain operations of algebra, and we show that if $S$ is a compact Hausdorff space, and if $C(S)$ has the supremum norm, then any multiplication which makes $C(S)$ a commutative Banach algebra renders its bidual commutative. We also give a constructive proof of the fact that if $G$ is an infinite locally compact abelian group, then the bidual of $L_{1}(G)$ is not commutative. In the second place, we prove that the projection to the base algebra of the radical in the bidual of a Banach algebra is precisely the radical of the base algebra. In addition, we determine that the weak*-closed maximal regular ideals in the biduals of a large class of commutative topological algebras (including commutative Banach algebras) are the weak*-closures in the biduals of closed maximal regular ideals in the base algebras. Furthermore, we show that if $T$ is a locally compact, noncompact Hausdorff space, then $C_{0}(T)$ is an ideal in its bidual if and only if $T$ is discrete; nevertheless, $C_{0}(T)$ can always be embedded in a regular ideal of its bidual. In the third place, we show that if $G$ is a locally compact abelian group, then the radical in the bidual of $L_{1}(G)$ is separable if and only if $G$ is finite.

We now amplify the above statements. The original discussions on the definition of the Arens multiplication occur in $[1,2]$, are extended in [11]. In [2] Arens gives a condition, involving weakly compact sets of the base algebra, which guarantees that the bidual of a commutative Banach algebra be commutative. He asks if there is a necessary condition of the same type. Our criterion in Section 3 arises from an attempt to answer his question, and goes as follows. Let $E=(E, \circ)$ be a topological algebra whose bidual has an Arens multiplication, and let $E^{*}$ be the dual of $E$. For $x^{*} \in E^{*}$, define $T_{x^{*}}: E \rightarrow E^{*}$ by $T_{x^{*}}(x)=x^{*} \circ x$, where $\left(x^{*} \circ x\right) y=x^{*}(x \circ y), x, y \in E$. 
Then $E^{* *}$ is commutative if and only if for each $x^{*} \in E^{*}, T_{x^{*}}$ is a weakly compact operator. Our related results follow from this characterization.

The result in Section 4 that the projection to the base algebra of the radical in the bidual of a Banach algebra is precisely the radical of the base algebra answers a conjecture of, and partial solution [4]. In Section 5 we discuss the radical in the bidual of $L_{1}(G)$, where $G$ is an abelian locally compact group. Civin and Yood [4] show that if $G$ is not discrete, or if $G$ is the group of integers, then the radical is infinite-dimensional. They conjecture that the radical is infinitedimensional for any discrete infinite abelian group. This is proved by Granirer as a by-product of an analysis of amenable semi-groups [9]. Our proof that the radical is always nonseparable uses a different approach. We conclude with a list of unresolved questions.

2. Preliminary definitions. Throughout the paper we will assume that $E$ is a locally convex (linear topological) space. If $E$ is a topological algebra, then $E$ is assumed to be an associative algebra such that the multiplication is separately continuous for the topology on $E$. For a set $S \subset E$, let $C S$ denote the complement of $S$ in $E$. The dual $E^{*}$ of a locally convex space $E$ consists of all continuous linear functions from $E$ into the complex numbers. Its members are denoted by $x^{*}, y^{*}$, etc. The topology on $E^{*}$ is normally the topology of uniform convergence on the bounded sets of $E$. The bidual $E^{* *}$ of $E$ is the dual of $E^{*}$, equipped with the topology of uniform convergence on bounded sets of $E^{*}$, and its members are denoted by $x^{* *}, y^{* *}$, etc. The weak- $\left(E^{* *}\right)$ and weak- $(E)$ topologies on $E^{*}$ are the topologies of uniform convergence on finite point sets of $E^{* *}$ and $E$ respectively. It is well-known that if we define the map $\Pi: E \rightarrow$ $E^{* *}$ by $\Pi(x) x^{*}=x^{*}(x)$, for all $x^{*} \in E^{*}$, then $E$ is embedded in $E^{* *}$, and $\Pi(E)$ is weak- $(E)$ dense in $E^{* *}$. If $F$ is a subset of the locally convex space $E$, then $F^{\perp}$ refers to all those elements of $E^{*}$ which annihilate $F$. If $E$ is an algebra, then it can be embedded in an algebra $E^{+}$with identity. Thus $E^{+}$is the cartesian product of $E$ with the complex numbers, under the product topology, where multiplication is defined as follows: for $x, y$ in $E$, and for $a, b$ complex, we have $(x, a)(y, b)=(x y+a y+b x, a b)$. A continuous, linear map $T$ from one locally convex space to another is called weakly compact if the image of any bounded set in the domain is relatively weakly compact in the range space. The adjoint of $T$ is written $T^{*}$.

If $E$ is a Banach algebra with multiplication $\circ$, then there exists on $E^{* *}$ an Arens multiplication, which we now construct. Although - will appear in several contexts as a bilinear function, we will always know on what spaces $\circ$ operates if we look at the elements to the left 
and to the right of $\circ$. First define on $\left(E^{*}, E\right)$ the bilinear operation $\circ$ by $\left(x^{*} \circ x\right) y=x^{*}(x \circ y)$, for all $y \in E$, where $x^{*} \in E^{*}$ and $x \in E$. Then $x^{*} \circ x \in E^{*}$. Next, define on $\left(E^{* *}, E^{*}\right)$ the operation $\circ$ by $\left(y^{* *} \circ x^{*}\right) x=$ $y^{* *}\left(x^{*} \circ x\right)$, for all $x \in E$, where $y^{* *} \in E^{* *}$ and $x^{*} \in E^{*}$. Then $y^{* *} \circ x^{*} \in$ $E^{*}$. Finally, define on $\left(E^{* *}, E^{* *}\right)$ the operation $\circ$ by $\left(x^{* *} \circ y^{* *}\right) x^{*}=$ $x^{* *}\left(x^{* *} \circ x^{*}\right)$, for all $x^{*} \in E^{*}$, where $x^{* *}, y^{* *} \in E^{* *}$. Then $\circ$ is a bilinear operation on $E^{* *}$ with values in $E^{* *}$, and $\circ$ makes $E^{* *}$ into a Banach algebra [2]. However, we can generalize the notion to a wide class of topological algebras, including those with jointly continuous multiplication [11]. Any topological algebra whose bidual has this Arens multiplication we call a topological algebra with bidual.

Standard references for algebraic and topological problems are [19] and [14], and for group algebras [20].

3. Commutativity in the bidual of a topological algebra with bidual. As we mentioned in Section 2, any topological algebra whose bidual has the Arens multiplication we call a topological algebra with bidual. In order to simplify the terminology, we make the following.

Definition 3.1. A topological algebra with bidual is bicommutative if and only if the bidual is commutative.

Now we give a necessary and sufficient condition that a commutative topological algebra with bidual be bicommutative. Our Theorem 3.4 is related to a question of Arens [2, p. 845], and might be the appropriate answer to his question. The inspiration for our proof comes from Theorem 4.2 of [12].

LeMma 3.2. Let $E$ and $F$ be locally convex spaces, and let $T$ : $E \rightarrow F$ be a continuous, linear map. Then $T$ is a weakly compact map if and only if $T^{*}$ is continuous with respect to the weak- $(F)$ and weak-( $\left.E^{* *}\right)$ topologies on $F^{*}$ and $E^{*}$ respectively.

Proof. This is part of Lemma 1.1 of [10].

Next, let $x^{*} \in E^{*}$, and define the operator $T_{x^{*}}: E \rightarrow E^{*}$ by the equation

$$
\left(T_{x^{*}}(x)\right) y=\left(x^{*} \circ x\right) y \text {, for all } x \text { and } y \text { in } E \text {. }
$$

Then $T_{x^{*}}(x)=x^{*} \circ x$, and $T_{x^{*}}$ is a continuous, linear map. Note that for each $y^{* *} \in E^{* *}, T_{x^{*}}^{*}\left(y^{* *}\right)=y^{* *} \circ x^{*}$, and for each $x^{* *}$ and $y^{* *}$ in $E^{* *},\left(T_{x^{*}}^{* *}\left(x^{* *}\right)\right) y^{* *}=x^{* *}\left(T_{x^{*}}^{*}\left(y^{* *}\right)\right)=\left(x^{* *} \circ y^{* *}\right) x^{*}$. We can state and prove: 
Lemma 3.3. Let $E$ be a commutative topological algebra with bidual. Then $E^{* *}$ is commutative if and only if for every $x^{*} \in E^{*}$, $T_{x^{*}}^{*}$ is continuous with respect to the weak- $\left(E^{*}\right)$ and the weak $\left(E^{* *}\right)$ topologies on $E^{* *}$ and $E^{*}$ respectively.

Proof. Assume that $E^{* *}$ is commutative. Let $x^{* *} \in E^{* *}$ and $x^{*} \in E^{*}$. If $\left(y_{\lambda}^{* *}\right)_{\lambda \in \Lambda}$ is a net which converges to $y^{* *}$ in the weak- $\left(E^{*}\right)$ topology, then

$$
\left(x^{* *} \circ y_{\lambda}^{* *}\right) x^{*}=y_{\lambda}^{* *}\left(x^{* *} \circ x^{*}\right) \underset{\lambda}{\longrightarrow} y^{* *}\left(x^{* *} \circ x^{*}\right)=\left(x^{* *} \circ y^{* *}\right) x^{*},
$$

whence $x^{* *}\left(T_{x^{*}}^{*}\left(y_{\lambda}^{* *}\right)\right) \underset{\lambda}{\longrightarrow} x^{* *}\left(T_{x^{*}}^{*}\left(y^{* *}\right)\right)$, and $T_{x^{*}}^{*}$ is continuous in the appropriate topologies. Conversely, assume that for each $x^{*} \in E^{*}, T_{x^{*}}^{*}$ is continuous for the weak- $\left(E^{*}\right)$ and the weak- $\left(E^{* *}\right)$ topologies on $E^{* *}$ and $E^{*}$ respectively. Let $x^{* *}$ and $y^{* *}$ be in $E^{* *}$. Assume that $\left(y_{\lambda}\right)_{\lambda \in A}$ is a net in $E$ such that $\left(\Pi\left(y_{\lambda}\right)\right)_{\lambda \in \Lambda}$ converges to $y^{* *}$ in the weak- $\left(E^{*}\right)$ topology. Then

$$
\left(\Pi\left(y_{\lambda}\right) \circ x^{* *}\right) x^{*}=\left(\Pi\left(y_{\lambda}\right)\right)\left(x^{* *} \circ x^{*}\right) \underset{\lambda}{\longrightarrow} y^{* *}\left(x^{* *} \circ x^{*}\right)=\left(y^{* *} \circ x^{* *}\right) x^{*} .
$$

But by hypothesis,

$$
\left(x^{* *} \circ \Pi\left(y_{\lambda}\right)\right) x^{*}=x^{* *}\left(T_{x^{*}}^{*}\left(\Pi y_{\lambda}\right)\right) \underset{\lambda}{\longrightarrow} x^{* *}\left(T_{x^{*}}^{*}\left(y^{* *}\right)\right)=\left(x^{* *} \circ y^{* *}\right) x^{*} .
$$

Since $E$ is commutative by assumption, it is easy to show that $\Pi\left(y_{\lambda}\right) \circ x^{* *}=x^{* *} \circ \Pi\left(y_{\lambda}\right)$, for each $\lambda$. Consequently $x^{* *} \circ y^{* *}=$ $y^{* *} \circ x^{* *}$, so that $E^{* *}$ is commutative.

THEOREM 3.4. Let $E$ be a commutative topological algebra with bidual. Then $E$ is bicommutative if and only if for each $x^{*} \in E^{*}$, $T_{x^{*}}$ is a weakly compact operator from $E$ to $E^{*}$.

Proof. The theorem follows directly from Lemmas 3.2 and 3.3.

We now give some reduction theory for commutativity in the biduals, and then exhibit a few examples. Let $E$ be a topological algebra with bidual. Let $V$ be a neighborhood of 0 in $E$, and let $p_{V}$ be the Minkowski functional on $V\left[13\right.$, p. 15]. Let $E_{V}$ be the normed algebra $E / p_{V}^{-1}(0)$, clothed in the quotient topology arising from the semi-normed topology induced by $p_{V}$ on $E$. If there is a subbasis $\left(V_{\lambda}\right)_{\lambda \in A}$ of neighborhoods of 0 in $E$ such that each $E_{V_{\lambda}}$ is a normed algebra, then $E$ is called a locally multiplicatively-convex algebra, and such algebras are topological algebras with biduals [11]. 
such that for each $\lambda \in A, E_{V_{\lambda}}$ is bicommutative, then $E$ is bicommutative.

Proof. Let $x^{*} \in E^{*}$. Then there is a $V_{\lambda}$ such that $x^{*}$ is bounded on $V_{\lambda}$, and hence $x^{*}$ can be identified with an element of $E_{V_{\lambda}}^{*}$. If $B$ is bounded in $E$, then $B$ is bounded (by identification) in $E_{V \lambda}$, so $\overline{\left(x^{*} \circ B\right)}$ is weak- $\left(E_{V \lambda}^{* *}\right)$ compact in $E_{V \lambda}^{*}$. But the topology on $E_{V \lambda}^{*}$ is stronger than the restricted topology of $E^{*}$, which means that $x^{*} \circ B$ is weak- $\left(E^{* *}\right)$ compact in $E^{*}$, and $E$ is bicommutative, by Theorem 3.4 .

By using Theorem 17.13 of [13] it is easy to show that the Cartesian product of bicommutative algebras is again bicommutative. On the other hand, we have:

THEOREM 3.6. If $E$ is a bicommutative algebra, and if $F$ is a subalgebra with the restriction topology, then $F$ is bicommutative.

Proof. Let $x_{F}^{*} \in F^{*}$, and let $B$ be an arbitrary bounded set in $F$. We must show that $\overline{x_{F}^{*} \circ B}$ is a weak- $\left(F^{* *}\right)$ compact set in $F^{*}$. First extend $x_{F}^{*}$ to $x_{E}^{*}$ in $E^{*}$ by the Hahn-Banach theorem. Since $B$ is bounded in $E$, the hypothesis tells us that $x_{E}^{*} \circ B$ is weak- $\left(E^{* *}\right)$ compact in $E^{*}$. Furthermore, there is a linear isomorphism between $\left(x_{E}^{*} \circ B\right)+E^{\perp}$ and $x_{F^{\prime}}^{*} \circ B$, where $x_{F}^{*}$ is just the function $x_{E}^{*}$ restricted to $F$. By Theorem 17.13 of [13], we have that $\overline{\left(x_{E}^{*} \circ B\right)}+F^{\perp}$ is a weak- $\left(E^{*} / F^{\perp}\right)^{*}$ compact set. However, the topology on $F^{*}$ is no stronger than the quotient topology of $E^{*}$ on $E^{*} / F^{\perp}$, so that $\overline{x_{F}^{*} \circ B}$ is therefore weak- $\left(F^{* *}\right)$ compact. Consequently $F$ is bicommutative.

We next consider those locally convex spaces $E$ and closed subspace $F$ such that any $B$ bounded in $E / F$ is contained in the image of a bounded set in $E$ under the canonical map $\varphi$ from $E$ to $E / F$. Included are the quotient spaces of all Banach spaces.

THEOREM 3.7. If $E$ is a bicommutative topological algebra and has the property just described, and if $F$ is a closed ideal in $E$, then $G=E / F$ is bicommutative.

Proof. Any $y^{*} \in G^{*}$ corresponds to $x^{*} \in F^{\perp}$ by the equation $y^{*}(u+F)=x^{*}(u)$, for all $u \in E$. Then $\left[y^{*} \circ(x+F)\right](z+F)=$ $\left(x^{*} \circ x\right) z$, so that $y^{*} \circ(x+F)$ is identified with $x^{*} \circ x$, where $x^{*} \in F^{\perp}$. Now define $T_{x^{*}}: E \rightarrow E^{*}$ by $T_{x^{*}}(x)=x^{*} \circ x$, and $T_{y^{*}}: G \rightarrow G^{*}$ by $T_{y^{*}}(x+F)=y^{*} \circ(x+F)$. Then $T_{x^{*}}$ and $T_{y^{*}}$ are continuous functions. Let $R$ be a closed, bounded set in $G$, and let $S$ be the bounded set in 
$E$, guaranteed by hypothesis to exist, such that $\varphi(S) \supseteqq R$. By identification in $E^{*}, T_{x^{*}}(S) \supseteqq T_{y^{*}}(R)$ and $\overline{T_{x^{*}}(S)}$ is weak-( $\left.E^{* *}\right)$ compact, by hypothesis. Thus $\overline{T_{y^{*}}(R)}$ is weak- $\left(E^{* *}\right)$ compact too. But then by Theorem 17.13 of $[13], \overline{T_{y^{*}}(R)}$ is weak $\left(G^{* *}\right)$ compact, so that $T_{y^{*}}$ is weakly compact. Since $y^{*} \in G^{*}$ was arbitrary, we have shown that $G$ is bicommutative.

EXAMPle 3.8. Let $S$ be a compact, Hausdorff space and let $C(S)$ be the Banach space of continuous, complex-valued functions on $S$, with supremum norm and any multiplication which makes $C(S)$ a commutative Banach algebra. As a Banach space $C^{*}(S)$ is weakly sequentially complete [7, IV. 13.22], so that by Theorem VI. 7.6 of [7], if $x^{*} \in C^{*}(S)$, then $T_{x^{*}}$ is a weakly compact operator. Therefore $C(S)$ is bicommutative. We can apply Theorem 3.5 to show that if $T$ is a completely regular space with $\mathscr{S}$ a collection of compact subsets of $T$ which generates $T$ is the sense of Michael (see [16, p. 76]), if $C(T)$ has the topology of uniform convergence on elements of $\mathscr{S}$, and finally if the multiplication on $C(T)$ is separately continuous and commutative, then $C(T)$ is bicommutative. We remark that Arens [1, p. 17] proved this for (real-valued) functions on a compact Hausdorff space, under pointwise multiplication.

If $G$ is an infinite abelian locally compact group, then $L_{1}(G)$ is not bicommutative [5, p. 535] and [4, Theorems 3.1, 3.12]. We next see how our criterion can be used to show that $L_{1}(G)$ is not bicommutative. First, however, we need a lemma due to M. Rajagopalan [21]:

LEMMA 3.9. If $G$ is a locally compact abelian group which is extremally disconnected, then $G$ is discrete.

Proof. If $G$ is extremally disconnected, then $G$ is an $F$ space [8, pp. 22 and 215]. On the other hand, since $G$ is extremally disconnected, there exists a compact open subgroup $H$ of $G$, and $H$ contains a compact metric subgroup $K$ which is infinite if $H$ is infinite [20, pp. 42 and 45]. But then $K$ is a compact metric subspace of an $F$ space, and thus has the discrete topology [8, p. 215]. Since $K$ is compact and discrete, $K$ is finite, whence $H$ is finite. But then $H$ is finite and open in $G$, which means that $H$, and hence $G$, has the discrete topology.

EXAMPLE 3.10. Let $G$ be a nondiscrete locally compact abelian group. Then $L_{1}(G)$ is not bicommutative.

Proof. By the lemma above, $G$ is not extremally disconnected, so in $G$ there are two disjoint open sets $S$ and $T$ such that $\bar{S} \cap \bar{T} \neq \varnothing$. 
Without loss of generality assume that $\bar{S}$ has finite Haar measure. Let $s \in \bar{S} \cap \bar{T}$. Let $\left(U_{\lambda}\right)_{\lambda \in \Lambda}$ be a neighborhood system of 0 in $G, U_{\lambda}$ compact, and let $\mu$ be Haar measure on $G$. Note that for each $\lambda$, $\mu\left(s+U_{\lambda} \cap \bar{S}\right) \neq 0$. For each $\lambda \in \Lambda$, let $W_{\lambda}$ be a neighborhood of 0 with the following properties: $W_{\lambda} \leqq U_{\lambda}, s+W_{\lambda} \cap \bar{S}=s+U_{\lambda} \cap \bar{S}$, and $\mu\left(s+W_{\lambda} \cap \bar{S}\right) \geqq(1 / 2) \mu\left(W_{\lambda}\right)$. In addition, let us define $b_{\lambda}=\mu\left(W_{\lambda}\right)$, for each $\lambda \in \Lambda$. For each $x^{*} \in L_{1}^{*}(G)$, let $h_{x^{*}} \in L_{\infty}(G)$ correspond to $x^{*}$. We then define $x_{0}^{*}$ by $h_{x_{0}^{*}}=\chi_{\bar{s}} \in L_{\infty}(G)$ and $x_{\lambda}=\left(1 / b_{\lambda}\right) \chi_{W_{\lambda}} \in L_{1}(G)$, for all $\lambda \in \Lambda$. Note that $\left\|x_{\lambda}\right\|=1$. Furthermore, for each $t \in G$,

$$
\begin{aligned}
h_{x_{0}^{*} \circ x_{\lambda}}(t) & =\int_{G} h_{x_{0}^{*}}(t+r) x_{\lambda}(r) d \mu(r) \\
& =\left(1 / b_{\lambda}\right) \int_{W_{\lambda}} h_{x_{0}^{*}}(t+r) d \mu(r) \\
& =\left(1 / b_{\lambda}\right) \mu\left(t+W_{\lambda} \cap \bar{S}\right) .
\end{aligned}
$$

If $t \in \mathrm{C} \bar{S}$, then since $G$ is regular, there is a $W_{\lambda_{0}}$ such that $t+W_{\lambda_{0}} \cap$ $\bar{S}=\phi . \quad$ Pick $\lambda_{1} \geqq \lambda_{0}$ such that $U_{\lambda_{1}} \cong W_{\lambda_{0}}$. Then $\left(t+U_{\lambda_{1}}\right) \cap \bar{S}=\phi$, so that if $\lambda \geqq \lambda_{1}$, then $0 \leqq h_{x_{0}^{*} \circ x_{\lambda}}(t)=\left(1 / b_{\lambda}\right) \mu\left[\left(t+W_{\lambda}\right) \cap \bar{S}\right] \leqq$ $\left(1 / b_{\lambda}\right) \mu\left[\left(t+U_{\lambda_{1}}\right) \cap \bar{S}\right]=0$. Thus on $C \bar{S},\left\{h_{x_{0}^{*} \circ \lambda_{\lambda}}\right\}_{\lambda \in A}$ converges pointwise to 0 . On the other hand, $h_{x_{0}^{*} \circ x_{\lambda}}(s)=\left(1 / b_{\lambda}\right) \mu\left(s+W_{\lambda} \cap \bar{S}\right) \geqq 1 / 2$, for all $\lambda \in \Lambda$. For each $\lambda \in \Lambda$, let $s_{\lambda} \in s+W_{\lambda} \cap C \bar{S}$, so that $s_{\lambda} \underset{\lambda}{\longrightarrow} s$ in the topology of $G$. Because of our preceding arguments, for any fixed $\lambda_{0}$ and any arbitrary $\lambda_{1}$, there is a $\lambda \geqq \lambda_{1}$ such that $\mid h_{x_{0}^{*} \circ x_{\lambda}}\left(s_{\lambda_{0}}\right)$ $h_{x_{0}^{*} \circ x_{\lambda}}(s) \mid \geqq 1 / 2$. But this means that $\left\{h_{x_{0}^{*} \circ x_{\lambda}}\right\}_{\lambda \in A}$ is not weakly sequentially compact [7, Theorem IV. 6.14]. Consequently by the EberleinSmulian theorem it is not relatively weakly compact, whence $T_{x_{0}^{*}}$ is not a weakly compact operator, and $L_{1}(G)$ is not bicommutative.

ExAMPLE 3.11. Let $G$ be a discrete locally compact abelian group with an element $g$ of infinite order. Then $L_{1}(G)$ is not bicommutative.

Proof. Let $h_{x^{*}}$ be the characteristic function of the set $(k g)_{k=0}^{\infty}$, and let $x_{m}$ be the characteristic function of the point $m g$, for $m=1,2, \cdots$. Then

$$
h_{x^{*} \circ x_{m}}(k g)=\sum_{n=0}^{\infty} x_{m}(n-k) g=\left\{\begin{array}{ll}
1 & \text { if } k \geqq-m \\
0 & \text { if } k<-m
\end{array}\right\} .
$$

Thus for any $m, \lim _{k \rightarrow-\infty} h_{x^{*} \circ x_{m}}(k g)=0$. On the other hand, if we fix $k_{1}, \cdots, k_{r}<0$, and if $-m<\inf \left(k_{1}, \cdots, k_{r}\right)$, then $h_{x^{*} \circ x_{m}}\left(k_{i} g\right)=1$ for $i=1, \cdots, r$. Consequently $\left(h_{x^{*} \circ x_{m}}\right)_{m=1}^{\infty}$ is not weakly sequentially compact [7, Theorem IV. 6.14], and hence $L_{1}(G)$ is not bicommutative.

For the infinite discrete locally compact abelian groups without any elements of infinite order, one can use the analysis of Section 5 
to show that if $e^{*}$ is the function identically 1 on $G$, then $T_{e^{*}}$ will not be weakly compact.

We also observe that one can easily show that $\ell_{1}$ is bicommutative under coordinate-wise multiplication or any inferior multiplication which makes $\iota_{1}$ into a topological algebra with bidual.

Finally, we should like to say a word about the question posed by Arens. He gives a condition [2], involving weakly compact sets of the base algebra, which guarantees that a commutative Banach algebra be bicommutative. The condition is: for every $\varepsilon>0$ there exists a weakly compact subset $B_{\varepsilon}$ of the unit ball $S$ such that for any $x \in S$ there is a $b_{x} \in B_{\varepsilon}$ for which if $y$ is any element of the Banach algebra, then $\left\|\left(x-b_{x}\right) y\right\| \leqq \varepsilon\|y\|$. Then Arens seeks a necessary condition also involving weakly compact sets of the base algebra. Although with his sufficient condition he can prove that $c_{0}$ (the sequences converging to 0 , with supremum norm and pointwise multiplication) is bicommutative, it appears to us that this condition is very restrictive and that there may exist no satisfactory necessary condition involving weakly compact sets in the base algebra. For we are able to show that if $S$ is a nonfinite, compact Hausdorff space, and if $C(S)$ is the Banach algebra of continuous, complex-valued functions on $S$, under pointwise multiplication and supremum norm, then $C(S)$ does not fulfill the Arens condition. The proof goes as follows. Let $s \in S$ be a fixed element such that there is a nonfinite net $\left(s_{\lambda}\right)_{\lambda \in \Lambda}$ converging to $s$. Note that $x \in C(S)$ implies that $\lim _{\lambda} x\left(s_{\lambda}\right)=$ $x(s)$, and for any finite collection $s_{\lambda_{1}}, \cdots, s_{\lambda_{n}}$, there is an $x_{0} \in C(S)$ such that $x_{0}(s)=0$ and $x_{0}\left(s_{\lambda i}\right)=1, i=1,2, \cdots, n$. If $B$ is any subset in $C(S)$ with the property, that if $x \in C(S)$ and $\|x\| \leqq 1$, then there is a $b_{x} \in B$ such that $\left\|x-b_{x}\right\| \leqq 1 / 4$, this means that for our $x_{0}$ we can find a $b_{x_{0}} \in B$ such that $\left|b_{x_{0}}\left(s_{\lambda i}\right)\right| \geqq 3 / 4, i=1,2, \cdots, n$, and $\left|b_{x_{0}}(s)\right| \leqq 1 / 4$. Thus $\left|b_{x_{0}}\left(s_{\lambda i}\right)-b_{x_{0}}(s)\right| \geqq 1 / 2, i=1,2, \cdots, n$. But then $B$ cannot be weakly compact [7, Theorem IV. 6.14], and Arens' criterion is not fulfilled. However $C(S)$ is bicommutative.

4. Ideals and the Radical in the Bidual. Let $E$ be a complete commutative topological algebra with bidual and continuous quasi-inversion. If $E$ is a locally multiplicatively-convex algebra, then it has these properties [11 and 16]. We give a characterization of the weak- $\left(E^{*}\right)$ closed maximal regular ideals in $E^{* *}$. Lemma 4.2 and Theorem 4.3 generalize Theorem 5.3 of [4] and Corollary 5.4 of [3] respectively.

Lemma 4.1. Let $M$ be a closed maximal regular ideal of $E$, and let $y$ be a right identity for $E$ modulo $M$. Let $M^{+}$be the closed algebra in $E^{+}$generated by $(M, 0)$ and $(y,-1)$. Then $M^{+}$is a closed maximal regular ideal in $E^{+}$, and $M^{+} \cap E=M$. 
Proof. Compare with $[15$, pp. 59, 60]. The proof is easy. The elements of $M^{+}$are of the form $(m+a y$, $-a)$, where $m \in M$ and $a$ is complex. Furthermore, $(0,1)$ is not in $M^{+}$, and $M^{+}$has the other properties mentioned.

LEMma 4.2. Let $M_{E}$ be a closed maximal regular ideal of $E$. Then the weali- $\left(E^{*}\right)$ closure $M$ of $\Pi\left(M_{E}\right)$ is a closed maximal regular two-sided ideal in $E^{* *}$.

Proof. We use here the continuity of quasi-inversion. For it guarantees that $M_{E}$ correspond to a complex-valued homomorphism on $E$, so that if $y$ is the identity for $E$ modulo $M_{E}$, then the linear space spanned by $M_{E}$ and $y$ is dense in $E$. We next recall that the second adjoint of a continuous homomorphism is a weak- $\left(E^{*}\right)$ continuous homomorphism [11, Remark 3.11]. We can then use verbatim the argument of Theorem 5.3 of [4] to conclude the proof.

THEOREM 4.3. Let E be a complete commutative topological algebra with bidual and continuous quasi-inversion. Let $M$ be a closed maximal regular ideal of $E^{* *}$. Then $M$ is weak-( $\left.E^{*}\right)$ closed if and only if $M$ is the weal- $\left(E^{*}\right)$ closure of some closed maximal regular ideal $M_{E}$ of $E$.

Proof. Lemma 4.2 shows that the image of the weak- $\left(E^{*}\right)$ closure of a closed maximal regular ideal in $E$ is a weak- $\left(E^{*}\right)$ closed maximal regular ideal of $E^{* *}$. Henceforth we take all closures in the weak- $\left(E^{*}\right)$ topology. Now assume that $M$ is a weak- $\left(E^{*}\right)$ closed maximal regular ideal in $E^{* *}$. Note that since $M$ is weak- $\left(E^{*}\right)$ closed and proper and since $\overline{\Pi(E)}=E^{* *}$, we have $M \nsupseteq \Pi(E)$. For the moment, assume that $E$ has an identity element. Let $M_{E} \subset E$ be defined by the equation $\Pi\left(M_{E}\right)=M \cap \Pi(E)$. Then $M_{E}$ is a closed maximal regular ideal in $E$. By Lemma $4.2, \overline{\Pi\left(M_{E}\right)}$ is a maximal regular ideal of $E^{* *}$. But $M \supseteqq \Pi\left(M_{E}\right)$ and $M$ is weak- $\left(E^{*}\right)$ closed, which means that $M \supseteqq \overline{\Pi\left(M_{E}\right)}$. The maximality of $\overline{\Pi\left(M_{E}\right)}$ yields the equation $M=\overline{\Pi\left(M_{E}\right)}$. This disposes of the cases in which $E$ has an identity element. We can therefore assume that $E$ does not have an identity element. Via Lemma 4.1 , embed $M$ in a weak- $\left(E^{+*}\right)$ closed maximal regular ideal $N$ of $E^{* *+} \cong E^{+* *}$. Using the preceding argument, we find a closed maximal regular ideal $N_{E^{+}}$in $E^{+}$whose weak-( $\left.E^{+*}\right)$ closure in $E^{+* *}$ is $N$. However, $N_{E^{+}}$corresponds to a continuous homomorphism from $E^{+}$to the complex numbers. The restriction of this homomorphism to $E$ is a continuous homomorphism on $E$ with kernel $N_{E^{+}} \cap E$. Call this kernel $M_{E}$. To show that $M_{E}$ is proper in $E$, we note that $M=N \cap E^{* *} \subset E^{* *}$, so that $\bar{M}_{E} \nsupseteq E^{* *}$, 
whence $M_{E}=N_{E^{+}} \cap E \subset E$. Then $M_{E}$ is a closed maximal regular ideal in $E$. Also, $\Pi\left(M_{E}\right)=\Pi\left(N_{E^{+}} \cap E\right) \subseteq N \cap \Pi(E)=M$. However, $\overline{\Pi\left(M_{E}\right)}$ is a maximal regular ideal in $E^{* *}$, by Lemma 4.2 , and since $M$ is weak- $\left(E^{*}\right)$ closed, we have the equality $\left.M=\overline{\Pi\left(M_{E}\right.}\right)$.

We now turn our attention to the radical in the bidual of an arbitrary Banach algebra. Later we will see that we are unduly restricting ourselves.

Lemma 4.4. Let $R_{E}$ and $R_{E^{+}}$denote the radicals of $E$ and $E^{+}$ respectively. Then by identification, $R_{E}=R_{E^{+}} \cap E$.

Proof. By Lemma 4.1, $R_{E} \supseteqq R_{E^{+}} \cap E$. On the other hand let $x$ be a fixed element in $R_{E}$. This is equivalent to the statement that if $y \in E$ and if $b$ is any complex number, then $(b+y) x$ is quasi-regular in $E$. Consider $(x, 0) \in E^{+}$. To show that $(x, 0) \in R_{E^{+}}$it suffices to show that for any $(y, a) \in E^{+}$and any complex number $c,[c+(y, a)]$ $(x, 0)$ is quasi-regular in $E^{+}$. But if we let $b=a+c$, then $[c+(y, a)](x, 0)=((b+y) x, 0)$, which is quasi-regular in $(E, 0)$, and hence in $E^{+}$. Thus $(x, 0) \in R_{E^{+}}$and we have $R_{E}=R_{E^{+}} \cap E$.

Lemma 4.5. Let $E$ have an identity element. Let $R_{E}$ and $R$ be the radicals of $E$ and $E^{* *}$ respectively. Then $R \cap \Pi(E)=\Pi\left(R_{E}\right)$.

Proof. Note that neither $E$ nor $E^{* *}$ is a radical algebra, since each has an identity element [4, Lemma 5.4]. Half the argument is already proved, since Lemma 5.7 of [4] shows that $R \cap \Pi(E) \leqq \Pi\left(R_{E}\right)$. On the other hand, let $M$ be a maximal regular left ideal in $E^{* *}$. If $M \supseteqq \Pi(E)$, then $M \cap \Pi(E) \supseteqq \Pi\left(R_{E}\right)$. But if $\quad(M \cap \Pi(E)) \subset \Pi(E)$ properly, then define $M_{E}$ in $E$ by the equation $\Pi\left(M_{E}\right)=M \cap \Pi(E)$, and note that $M_{E}$ is once again a maximal regular left ideal in $E$. Consequently $M \cap \Pi(E)=\Pi\left(M_{E}\right) \supseteqq \Pi\left(R_{E}\right)$. Therefore, $\quad R \cap \Pi(E)=$ $\cap\left\{[M \cap \Pi(E)]: M\right.$ a maximal regular left ideal in $\left.E^{* *}\right\} \supseteqq \Pi\left(R_{E}\right)$. Therefore $R \cap \Pi(E)=\Pi\left(R_{E}\right)$, which completes the proof.

Theorem 4.6. Let $E$ be any Banach algebra. Let $R_{E}$ and $R$ be the radicals of $E$ and $E^{* *}$ respectively. Then $R \cap \Pi(E)=\Pi\left(R_{E}\right)$.

Proof. Let $R_{E^{+}}, R_{E^{+* *}}$, and $R_{E^{* *+}}$ be the radicals of $E^{+}, E^{+* *}$, and $E^{* *+}$ respectively. Because $E^{+* *}$ and $E^{* *+}$ are naturally algebraically and topologically isomorphic, we can identify $R_{E^{+* *}}$ with $R_{E^{* *+}}$. By Lemma 4.4, $R_{E^{* *}} \cap E^{* *}=R$ and also $R_{E^{+}} \cap E=R_{E}$. By Lemma 4.5, $\Pi\left(R_{E^{+}}\right)=R_{E^{+* *}} \cap \Pi\left(E^{+}\right)$. Using our identifications, we 
obtain $\Pi\left(R_{E}\right)=\Pi\left(R_{E^{+}}\right) \cap \Pi(E)=\left[R_{E^{+* *}} \cap \Pi\left(E^{+}\right)\right] \cap \Pi(E)=R_{E^{* *}+} \cap \Pi(E)=$ $R \cap \Pi(E)$, which means that $R \cap \Pi(E)=\Pi\left(R_{E}\right)$.

Theorem 4.6 answers a conjecture of Civin and Yood [4, p. 866]. We remark that the only property of Banach algebras we specifically needed was that the radical in $E^{* *}$ was the intersection of the closed regular maximal left ideals of $E^{* *}$, which happens also if $E^{* *}$ is a commutative, complete locally multiplicatively-convex algebra $[16, \mathrm{p}$. 58]. The operator theory used in Lemma 5.7 of [4] is valid more generally.

We next give a new proof of Theorem 3.3 of [3], with the aid of:

Lemma 4.7. Assume that $E$ is a commutative Banach algebra with an approximate identity but no identity. Let $e^{* *}$ be a right identity in $E^{* *}$, which exists by Lemma 3.8 of [4]. Let there exist $a$ bounded $\left(x_{n}^{*}\right)_{n=1}^{\infty}$ in $E^{*}$ such that $e^{* *}\left(x_{n}^{*}\right)=1$ for all $n$, but such that $\left\|x_{n}^{*} \circ x\right\| \underset{n}{\longrightarrow} 0$ for each $x \in E$. Then $\Pi(E)$ is contained in a left regular ideal of $E^{* *}$.

Proof. Let $y^{* *} \in E^{* *}$. Then $\left\|x_{n}^{*} \circ x\right\| \underset{n}{\longrightarrow} 0$ implies that $y^{* *}\left(x_{n}^{*} \circ x\right) \underset{n}{\longrightarrow} 0$. Consequently $\left(\Pi(x) \circ y^{* *}\right) x_{n}^{*} \underset{n}{\longrightarrow} 0$. If $\left(x_{i}\right)_{i=1}^{m} \subset E$ and $\left(y_{i}^{* *}\right)_{i=1}^{m} \subset E^{* *}$, then $\sup _{n}\left|\left\{\left[\sum_{i=1}^{m}\left(\Pi\left(x_{i}\right) \circ y_{i}^{* *}\right)\right]-e^{* *}\right\} x_{n}^{*}\right| \geqq 1$, since $e^{* *}\left(x_{n}^{*}\right)=1$ for each $n$ by hypothesis. Therefore $e^{* *}$ is not in the closure of the right ideal generated by $\Pi(E)$, and thus not in the closed left ideal, because $\Pi(E)$ is in the center of $E^{* *}$ [4, Lemma 3.9]. Since $E^{* *}$ has a right identity, this latter ideal is regular.

THEOREM 4.8. Let $G$ be a locally compact, nondiscrete, abelian group. Then $\Pi\left(L_{1}(G)\right)$ can be embedded in a left regular ideal of $L_{1}^{* *}(G)$.

Proof. (The multiplication in $L_{1}(G)$ is assumed to be convolution.) Let $x \in L_{1}(G)$ and $y^{* *} \in L_{1}^{* *}(G)$ be arbitrary elements. Let $\left(F_{n}\right)_{n=1}^{\infty}$ be a sequence of measurable neighborhoods of the identity in $G$ such that $\mu\left(F_{n}\right) \underset{n}{\longrightarrow} 0$, where $\mu$ is Haar measure. For $x^{*} \in L_{1}^{*}(G)$, let $h_{x^{*}}$ be the $L_{\infty}(G)$ function corresponding to $x^{*}$. Also, let $x \in L_{1}(G)$. Then the Fubini theorem yields

$$
h_{x^{*} \circ x}(s)=\int_{\theta} h_{x^{*}}(t) x(t-s) d \mu(t), \quad s \in G .
$$

Let $h_{x_{n}^{*}}$ be the characteristic function of $F_{n}$, for all $n$. Then $\left\|h_{x_{n}^{*} \circ x}\right\| \underset{n}{\longrightarrow} 0$, since $\mu\left(F_{n}\right) \underset{n}{\longrightarrow} 0$, by Theorem III. 2.20 of [7] and the invariance of $\mu$. Now let $m_{y^{* *}}$ be the finitely additive measure on $G$ 
which corresponds to $y^{* *} \in L_{1}^{* *}(G)$. Since the norm of $y^{* *}$ is finite, we have

$$
\left|y^{* *}\left(x_{n}^{*} \circ x\right)\right| \leqq \int_{G}\left\|h_{x_{n}^{*} \circ x}\right\| d\left|m_{y^{* *}}(t)\right|=\left\|x_{n}^{*} \circ x\right\|\left\|y^{* *}\right\| \underset{n}{\longrightarrow} 0 .
$$

On the other hand, since $e^{* *}$ is a weak- $\left(L_{1}^{*}(G)\right)$ cluster point of the elements of an approximate identity, the finitely additive measure corresponding to it has measure 1 on any set which is a neighborhood of the identity in $G$, i.e., $e^{* *}\left(x_{n}^{*}\right)=1$ for all $n$. Therefore the hypotheses of Lemma 4.7 are satisfied, and $\Pi\left(L_{1}(G)\right)$ can be embedded in a left regular ideal of $L_{1}^{* *}(G)$.

Let $T$ be a locally compact, Hausdorff space which is not compact. Let $C_{0}(T)$ be the Banach algebra under pointwise multiplication and supremum norm of the continuous, complex-valued functions on $T$ which vanish at infinity. Then we have:

Theorem 4.9. $\Pi\left(C_{0}(T)\right)$ is an ideal in $C_{0}^{* *}(T)$ if and only if $T$ is discrete.

Proof. Let $T$ be discrete. The dual of $C_{0}(T)$ is $\ell_{1}(T)$ and the bidual is $\ell_{\infty}(T)$. Let $y \in C_{0}(T)$ and let $\chi_{t}$ be the characteristic function of $t$, for any $t \in T$. Then $\chi_{t} \in C_{0}^{*}(T)$. If $h \in C_{0}^{* *}(T)$, then $(\Pi(y) \circ h) t=h\left(\chi_{t} \circ y\right)=h(t) y(t)$, so that since $h$ is uniformly bounded on $T, \Pi(y) \circ h$ vanishes at infinity, and therefore $\Pi(y) \circ h \in \Pi\left(C_{0}(T)\right)$. From Theorem 3.6 and Example 3.8 we find that $\Pi\left(C_{0}(T)\right)$ is an ideal in $C_{0}^{* *}(T)$. Next, assume that $T$ is not discrete. We separate the argument into two parts. First, assume that if $V$ is a compact subset of $T$ then $V$ carries no atomless regular Borel measure. For such $V$, it is known that $C^{*}(V)$ is equivalent to $\iota_{1}(V)[18, \mathrm{p} .214]$. It follows by easy calculation that $C_{0}^{*}(T)$ is equivalent to $\iota_{1}(T)$, so that $C_{0}^{* *}(T)$ is equivalent to $\ell_{\infty}(T)$. Let $t_{0} \in T$ have an infinite neighborhood system $\left(U_{\lambda}\right)_{\lambda \in \Lambda \text {. }}$ Let $h\left(t_{\lambda}\right)=1, t_{\lambda} \in U_{\lambda}, t_{\lambda} \neq t_{0}, \lambda \in \Lambda$, and $h(t)=0$, all other $t \in T$. Then $h \in \ell_{\infty}(T)$. If $y \in C_{0}(T)$ is such that $y\left(t_{0}\right)=1$, then we have $\lim _{\lambda}(\Pi(y) \circ h) t_{\lambda}=\lim _{\lambda} y\left(t_{\lambda}\right) h\left(t_{\lambda}\right)=1$ and $(\Pi(y) \circ h) t_{0}=y\left(t_{0}\right) h\left(t_{0}\right)=$ 0 , so that $(\Pi(y) \circ h) \notin \Pi\left(C_{0}(T)\right)$, and therefore $\Pi\left(C_{0}(T)\right)$ is not an ideal in $C_{0}^{* *}(T)$. On the other hand assume that there is a compact subset $V$ in $T$ with an atomless regular Borel measure. This measure corresponds to an element $x^{*} \in C_{0}^{*}(T)$, since by the StoneWeierstrass theorem, $C_{0}(T)$ restricted to $V$ is norm-dense in $C(V)$. Furthermore we can indeed find a $y \in C_{0}(T)$ such that $\left\|x^{*} \circ y-x^{*}\right\| \leqq$ 1/2. For $t \in T$, let $x_{t}^{*}$ be the point mass at $t$, and note that $x^{*}$ is not in the norm closure of the span of $\left(x_{t}^{*}\right)_{t \in T}$, since $x^{*}$ is atomless. Pick $w^{* *} \in C_{0}^{* *}(T)$ such that $w^{* *}$ vanishes on each $x_{t}^{*}$, for $t \in T$, but such that $w^{* *}\left(x^{*}\right)=1$. Without loss of generality let $\left\|w^{* *}\right\|=\left\|x^{*}\right\|=1$. 
Then

$$
\left|\left(w^{* *} \circ x^{*}\right) y\right|=\left|w^{* *}\left(x^{*}\right)+\left\{w^{* *}\left[\left(x^{*} \circ y\right)-x^{*}\right]\right\}\right| \geqq 1-1 / 2=1 / 2,
$$

so that $w^{* *} \circ x^{*} \neq 0$. If $\Pi(y) \circ w^{* *}=\Pi(z)$ for some $z \in C_{0}(T)$, then $z(t)=[\Pi(z)] x_{t}^{*}=\left[\Pi(y) \circ w^{* *}\right] x_{t}^{*}=w^{* *}\left(x_{t}^{*} \circ y\right)=y(t) w^{* *}\left(x_{t}^{*}\right)=0, t \in T$, so $z=0$. On the other hand, $x^{*}(z)=\left[\Pi(y) \circ w^{* *}\right] x^{*}=\left(w^{* *} \circ x^{*}\right) y \neq 0$ by the above calculation, whence $z \neq 0$. This contradiction proves that in our second case, $I\left(C_{0}(T)\right)$ is not an ideal in $C_{0}^{* *}(T)$.

Nevertheless, we do have the following:

THEOREM 4.10. For any locally compact, noncompact Hausdorff $T, \Pi\left(C_{0}(T)\right)$ is contained in a proper regular ideal of $C_{0}^{* *}(T)$.

Proof. If $T$ is discrete, then the statement follows from Theorem 4.9. In any case, $C_{0}(T)$ has an approximate identity, and therefore $C_{0}^{* *}(T)$ has a right identity $e^{* *}$ [4, Lemma 3.8]. Hence, for each point mass $x_{t}^{*}$, we have $e^{* *}\left(x_{t}^{*}\right)=1$. Pick $v^{* *} \in C_{0}^{* *}(T)$ and $x \in C_{0}(T)$. Then

$$
\left|\left(I I(x) \circ v^{* *}\right) x_{t}^{*}\right| \leqq\left\|v^{* *}\right\|\left\|x_{t}^{*} \circ x\right\|=\left\|v^{* *}\right\||x(t)|, \quad t \in T .
$$

Since $x$ vanishes at infinity, this means that $\left\|\Pi(x) \circ v^{* *}-e^{* *}\right\| \geqq 1$, so that $e^{* *}$ is far away from linear combination of elements like $\Pi(x) \circ v^{* *}$, and hence is far away from the ideal in $C_{0}^{* *}(T)$ generated by $I\left(C_{0}(T)\right)$. However, by Theorem 3.6 and Example $3.8, C_{0}^{* *}(T)$ is commutative. Consequently the ideal generated by $\Pi\left(C_{0}(T)\right)$ is regular.

5. Nonseparability of the radical in $L_{1}^{* *}(G)$. In this section we give a proof that for any abelian locally compact group, the radical $R$ of $L_{1}^{* *}(G)$ is not only infinite-dimensional, but even nonseparable. That proof we divide into three parts. All measures are assumed only finitely additive.

LEMMA 5.1. Let $G$ be an infinite discrete abelian group. Then there is a bounded, positive translation-invariant measure on $G$.

\section{Proof. See Day [6] and von Neumann [17].}

As we shall see, translation-invariant measures on $G$ give rise to elements of the radical in $L_{1}^{* *}(G)$. One might hope that we could produce a great many such positive translation-invariant measures as we have just done, by merely picking various $m$ 's. However, there seems to be no guarantee by the Kakutani-Markov theorem that the various resulting fixed points would be different from one another. So we proceed along a slightly different avenue. 
Lemma 5.2. If $G$ is not discrete, then $R$ is nonseparable.

Proof. By Lemma 3.9, $G$ cannot be extremally disconnected. Thus there exist disjoint open sets $U, V$ in $G$ such that $\bar{U} \cap \bar{V} \neq \phi$. Without loss of generality assume that $0 \in \bar{U} \cap \bar{V}$. Let $\left(W_{\lambda}\right)_{\lambda \in A}$ be a neighborhood system for 0 consisting of compact sets. Let $T_{\lambda}=$ $W_{\lambda} \cap \bar{U}$, for each $\lambda \in \Lambda$. By judicious selection we can produce sequences $\left(x_{n}\right)_{n=1}^{\infty} \subset G$ and $\left(\lambda_{n}\right)_{n=1}^{\infty} \subset \Lambda$ such that if $n \neq m$, then $\left(W_{\lambda_{n}}+x_{n}\right) \cap\left(W_{\lambda_{m}}+x_{m}\right)=\phi$. Let $\alpha$ be an infinite subsequence of the positive integers, and let

$$
\left.h_{\alpha}=\chi_{n_{i} \in \alpha}^{\cup\left(T_{\lambda_{n}}+x\right.} n_{n_{i}}\right) \cdot
$$

Then $\left\|h_{\alpha}-f\right\|_{\infty} \geqq 1$, for any function $f$ which is continuous on $G$, by prescription of the $T_{\lambda_{n}}+x_{n}$. Furthermore, if $\beta$ is a different infinite subsequence of positive integers, then $\left\|h_{\alpha}-h_{\beta}\right\|=1$, and $\left\|h_{\alpha}-h_{\beta}-f\right\|_{\infty} \geqq 1$ for any $f$ continuous on $G$. However, there are an uncountable number of infinite subsequences of positive integers. Let $D(G)$ denote the subspace of $L_{1}^{*}(G)$ consisting of continuous, bounded functions. Then $L_{1}^{*}(G) / D(G)$ with the quotient norm is nonseparable, by our previous statements. Thus its dual, which can be represented as the subspace $[D(G)]^{\perp}$ of $L_{1}^{* *}(G)$, is nonseparable [7, Lemma II. 3.16). However, any measure in $L_{1}^{* *}(G)$ which vanishes on $D(G)$ is in the radical $R$ of $L_{1}^{* *}(G)$ [4, Theorems 3.10, 3.12, and Corollary 3.11]. Thus $R$ is nonseparable.

Lemma 5.3. If $G$ is discrete and has an element of infinite order, then $R$ is nonseparable provided $G$ is abelian.

Proof. Let $g \in G$ have infinite order, and let $N=\{n g: n$ an integer\}. Let $S$ be the shift operator on $L_{\infty}(N)$, i.e., $(S x) n g=$ $x(n-1) g$, all $n g \in N$ and all $x \in L_{\infty}(N)$. Then a routine check shows that $(I-S) L_{\infty}(N)$ consists of all functions $x \in L_{\infty}(N)$ such that $\left\{\left|\sum_{n=0}^{k} x(n g)\right|\right\}_{k=-\infty}^{\infty}$ is uniformly bounded. Also, $(I-S) L_{\infty}(N)$ is a linear space, and if $E$ is an infinite subset of integers, then for any $h \in(I-S) L_{\infty}(N),\left\|\chi_{E}-h\right\| \geqq 1$. Let $\overline{(I-S) L_{\infty}(N)}$ have closure taken in the supremum norm. Let $D=L_{\infty}(N) \overline{(I-S) L_{\infty}(N)}$, when $D$ has the quotient norm. Then as in Lemma 5.2, $D$ is nonseparable, which means once again that $D^{*}$ is also nonseparable. Also, $D^{*}$ may be regarded as a subspace of $L_{1}^{* *}(N)$. By the definition of $(I-S) L_{\infty}(N)$, if $m \in L_{1}^{* *}(N)$ is such that $m$ annihilates $(I-S) L_{\infty}(N)$, then $m$ is a translation-invariant measure on $N$. Thus the elements of $D^{*}$ are $N$-translation-invariant measures. By Lemma 5.1, $G$ has a translationinvariant measure $\mu$ of norm 1 . Now if $m \in D^{*}$, and if $M$ is the 
measure defined on $G$ by $M(E)=m(E \cap N)$ for any set $E$ in $G$, then $\mu \circ M$ is a translation-invariant measure in $L_{1}^{* *}(G)$, and $\|\mu \circ M\|=$ $\|m\|$ [5, Theorem 7.2]. Thus distinct measures on $N$ produce distinct measures on $G$. Let such invariant measures $\mu \circ M$ be denoted by $v$, and pick one specific one $v_{0}$. Let $e$ be the function identically 1 on $G$. For any of our $v$, let $a_{v}$ be a complex number such that $\left(v_{0}-a_{v} v\right) e=0$. Since $v_{0}-a_{v} v$ is once again translation-invariant, the left ideal in $L_{1}^{* *}(G)$ generated by $v_{0}-a_{v} v$ consists of the scalar multiples of $v_{0}-a_{v} v$, and thus $\left(v_{0}-a_{v} v\right) \circ\left(v_{0}-a_{v} v\right)=\left[\left(v_{0}-a_{v} v\right)(e)\right]$ $\left(v_{0}-a_{v} v\right)=0$, so that the ideal is nilpotent [5, Theorem 6.1]. Consequently $v_{0}-a_{v} v \in R\left[19\right.$, Theorem 2.3.5]. The nonseparability of $D^{*}$ then ensures the nonseparability of $R$.

LEMmA 5.4. If $G$ is an infinite abelian, discrete and contains no element of infinite order, then $R$ is nonseparable.

Proof. We refine a procedure of Day for creating invariant measures [5, Lemma 7.4]. Let $H=\cup_{n=1}^{\infty} H_{n} \leqq G$ where for each $n$, $H_{n}$ is a finite subgroup of $G$ and $H_{n} \subset H_{n+1}$. Let $p_{n}$ be the number of elements in $H_{n}$, and assume without loss of generality that $p_{n+1} \geqq$ $10 p_{n}$, for all $n$. Next we collect infinite sequences of positive integers $\left(\left(n_{k}\right)_{k=1}^{\infty}\right)_{n=1}^{\infty}$, such that if $\left(n_{k}\right)_{k=1}^{\infty}$ and $\left(m_{j}\right)_{j=1}^{\infty}$ are two different sequences, then $n_{k} \neq m_{j}$, for all $k, j$. For any $n$ and $k$, define the measure $m_{n_{k}}$ by

$$
m_{n_{k}}(g)=\left\{\begin{array}{l}
1 / p_{n_{k}} \text { if } g \in H_{n_{k}} \\
0 \text { all other } g \in H
\end{array}\right\}
$$

Then $m_{n_{k}} \in L_{1}^{*}(H)$. Now, for each $n$, define $x_{n} \in L_{\infty}(H)$ as follows:

$$
x_{n}=\chi_{k=1}^{\infty}\left(\boldsymbol{H}_{n k} \cap \mathrm{C} \boldsymbol{H}_{n k-1}\right)
$$

Then for all $k, m_{n_{k}}\left(x_{n}\right) \geqq .9$, whereas if $q \neq n$, then $m_{q_{j}}\left(x_{n}\right) \leqq .1$ for all $j$. For each $n$, let $m_{n}$ be a weak- $\left(L_{1}^{*}(H)\right)$ cluster point of $\left(m_{n_{k}}\right)_{k=1}^{\infty}$. Note that $m_{n}\left(x_{n}\right) \geqq .9$, while $m_{q}\left(x_{n}\right) \leqq .1$ if $q \neq n$. Consequently if $q \neq n$, then $\left\|m_{n}-m_{q}\right\| \geqq 1 / 2$, and for each $n$, $\left\|m_{n}\right\| \leqq 1$. Now we embark on showing that for any $n, m_{n}$ is translation-invariant on $H$. Let the shift $L_{r}$ on $H$ be defined for any $r \in H$ by $\left(L_{r} x\right) h=x(r h)$, all $h \in H$, all $x \in L_{\infty}(H)$. Then $m_{n}$ is translation-invariant on $H$ if and only if $L_{r}^{*} m_{n}=m_{n}$, for all $r \in H$. Momentarily, let $k$ be an arbitrary positive integer, and let $r \in H_{n_{k}}$. Then $x^{*} \in L_{1}^{*}(H)$ implies that since $H_{n_{k}}$ is a group, we have

$$
\left(L_{r}^{*} m_{n_{k}}-m_{n_{k}}\right) x^{*}=\sum_{h \in H_{n_{k}}}\left(1 / p_{n_{k}}\right) x^{*}(r h)-\sum_{h \in B_{n_{k}}}\left(1 / p_{n_{k}}\right) x^{*}(h)=0 .
$$


Thus if $r \in H_{n_{k}}$ then $L_{r}^{*} m_{n_{k}}=m_{n_{k}}$ and $m_{n_{k}}$ is translation-invariant on $H_{n_{k}}$. Next, if $r \in H$, then there is a $k_{0}$ such that $r \in H_{n_{k}}$ for all $k \geqq k_{0}$. Let $x^{*} \in L_{1}^{*}(H)$. Since $m_{n}$ is a weak- $\left(L_{1}^{*}(H)\right)$ cluster point of $\left(m_{n_{k}}\right)_{k=1}^{\infty}$, we can extract a subsequence $\left(q_{i}\right)_{i=1}^{\infty}$ from $\left(m_{k}\right)_{k=1}^{\infty}$ such that $m_{n}\left(L_{r} x^{*}\right)=\lim _{i} m_{q_{i}}\left(L_{r} x^{*}\right)$ and $m_{n}\left(x^{*}\right)=\lim _{i} m_{q_{i}}\left(x^{*}\right)$. Thus

$$
\left(L_{r}^{*} m_{n}\right) x^{*}=m_{n}\left(L_{r} x^{*}\right)=\lim _{i} m_{q_{i}}\left(L_{r} x^{*}\right)=\lim _{i} m_{q_{i}}\left(x^{*}\right)=m_{n}\left(x^{*}\right),
$$

so that $m_{n}$ is translation-invariant on $H$. Finally we show that from the invariant measures $\left(m_{n}\right)_{n=1}^{\infty}$ we can find a nondenumerable collection of translation-invariant measures on $H$ which are far apart from each other. To begin with, let $A_{n}=\cup_{k=1}^{\infty} H_{n_{k}} \cap \mathrm{CH}_{n_{k}-1}$ for each $n$. Note that if $m \neq n$, then $A_{n} \cap A_{m}=\phi$. Let $\mathscr{F}$ be an ultrafilter of the collection of positive integers. For each $F \in \mathscr{F}$, let $k_{F} \in F$, and define $m_{F}$ to be $m_{k_{F}}$. Note that $m_{F}$ is a translation-invariant measure as described above. Using the inclusion ordering in filters, we obtain a generalized sequence $\left(m_{F}\right)_{F \in \mathscr{F}}$ of translation-invariant measures in the unit ball of $L_{1}^{* *}(H)$, so they have a weak- $L_{1}^{*}(H)$ cluster point $m_{\mathscr{F}}$. Indeed, $m_{\mathscr{F}}$ is a translation-invariant measure [5, p. 520]. If $\mathscr{F}_{1}$ and $\mathscr{F}_{2}$ are different ultrafilters, then there is a set $U \in \mathscr{F}_{1}$ such that $c U \in \mathscr{F}_{2}$. Let $A_{U}=\cup_{n \in U} A_{n}$. By construction, $m_{\mathscr{F}_{1}}\left(\chi_{A_{U}}\right) \geqq .9$, while $m_{\mathscr{F}_{2}}\left(\chi_{\mathbb{A}_{U}}\right) \leqq .1$, so that $\left\|m_{\mathscr{F}_{1}}-m_{\mathscr{F}_{2}}\right\| \geqq 1 / 2$. But there are an uncountable number of ultrafilters on the positive integers. Thus we can find that many translation-invariant measures on $H$ at least a distance $1 / 2$ from one another. Now use the argument of the last portion of Lemma 5.3 to show that $R$ is nonseparable.

THEOREM 5.5. If $G$ is a locally compact abelian group, then $R$ is separable if and only if $G$ is finite.

Proof. If $G$ is finite, then $L_{1}^{* *}(G)$ is finite-dimensional. Otherwise, use Lemmas 5.2, 5.3, and 5.4.

6. Unsolved questions.

1. Let $S$ be the unit disk in the complex plane, and let $E$ be the subspace of $C(S)$ consisting of all functions analytic in the interior of $S$. Is $E$ bicommutative under any commutative multiplication which makes $E$ a Banach algebra?

2. Is Theorem 4.6 true for any topological algebra with bidual?

3. If $G$ is an infinite, discrete, abelian locally compact group, does the radical in $L_{1}^{* *}(G)$ necessarily contain elements which are not translation-invariant measures?

4. If $G$ is an infinite, nonabelian locally compact group, is the radical in $L_{1}^{* *}(G)$ nonzero? 
We are indebted to W. G. Bade for valuable discussions, and to the referee for his comments.

\section{REFERENCES}

1. R. F. Arens, Operations induced in function classes, Monatshefte für Math. u. Physik. 55 (1951), 1-19.

2. - The adjoint of a bilinear operation, Proc. Amer. Math. Soc. 2 (1951), 839-848.

3. P. Civin, Ideals in the second conjugate algebra of a group algebra, Math. Scand. 11 (1962), 161-174.

4. P. Civin and B. Yood, The second conjugate space of a Banach algebra as an algebra, Pacific J. Math. 11 (1961), 847-870.

5. M. M. Day, Amenable semigroups, Illinois J. of Math. 1 (1957), 509-544.

6. — Fixed-point theorems for compact convex sets, Illinois J. of Math. 5 (1961), 585-590.

7. N. Dunford and J. T. Schwartz, Linear operators, part I: general theory, Interscience, New York, 1958.

8. L. Gillman and M. Jerison, Rings of continuous functions, Van Nostrand, Princeton, 1960.

9. E. Granirer, $A$ theorem on amenable semigroups, Trans. Amer. Math. Soc. 111 (1964), 367-379.

10. A. Grothendieck, Sur les applications lineaires faiblement compactes d'espaces du type $C(K)$, Canadian J. Math 5 (1953), 129-173.

11. S. L. Gulick, The bidual of a locally multiplicatively-convex algebra, Pacific J. Math. 16 (1966), 1-26.

12. H. M. Kamowitz, Cohomology groups of commutative Banach algebras, Trans. Amer. Soc. 102 (1962), 352-372.

13. J. L. Kelley and I. Namioka and co-authors, Linear topological spaces, Van Nostrand, New York, 1963.

14. G. M. Köthe, Topologische lineare Räume part I, Springer, Berlin, 1960.

15. L. H. Loomis, An introduction to abstract harmonic analysis, Van Nostrand, Princeton, 1953.

16. E. A. Michael, Locally multiplicatively-convex topological algebras, Memoirs Amer. Math. Soc. 11 (1952).

17. J. von Neumann, Zur allgemeinen Theorie des Masses, Fund. Math. 13 (1929), 73-116.

18. A. Pelczynski and Z. Semadeni, Spaces of continuous functions (III), Studia Math. 18 (1959), 211-222.

19. C. E. Rickart, General theory of Banach algebras, Van Nostrand, Princeton, 1960.

20. W. Rudin, Fourier analysis on groups, Interscience, New York, 1962.

21. M. Rajagopalan, Fourier transform in locally compact groups, Acta Szeged 25 (1964), 86-89.

Received February 26, 1965.

UNIVERSITY OF PENNSYLVANIA 



\section{PACIFIC JOURNAL OF MATHEMATICS}

H. SAMELSON

Stanford University Stanford, California

R. M. BLUMENTHAL

University of Washington

Seattle, Washington 98105

\section{EDITORS}

\author{
*J. DugundJI \\ University of Southern California \\ Los Angeles, California 90007 \\ RICHARD ARENS \\ University of California \\ Los Angeles, California 90024
}

\section{ASSOCIATE EDITORS}

E. F. BeCKENBACH

B. H. NEUMANN

F. WOLF

K. YosidA

\section{SUPPORTING INSTITUTIONS}

UNIVERSITY OF BRITISH COLUMBIA

CALIFORNIA INSTITUTE OF TECHNOLOGY

UNIVERSITY OF CALIFORNIA

MONTANA STATE UNIVERSITY

UNIVERSITY OF NEVADA

NEW MEXICO STATE UNIVERSITY

OREGON STATE UNIVERSITY

UNIVERSITY OF OREGON

OSAKA UNIVERSITY

UNIVERSITY OF SOUTHERN CALIFORNIA
STANFORD UNIVERSITY

UNIVERSITY OF TOKYO

UNIVERSITY OF UTAH

WASHINGTON STATE UNIVERSITY

UNIVERSITY OF WASHINGTON

AMERICAN MATHEMATICAL SOCIETY CHEVRON RESEARCH CORPORATION TRW SYSTEMS

NAVAL ORDNANCE TEST STATION 


\section{Pacific Journal of Mathematics}

Vol. 18, No. 1

March, 1966

Edward Joseph Barbeau, Semi-algebras that are lower semi-lattices ...... 1

Steven Fredrick Bauman, The Klein group as an automorphism group

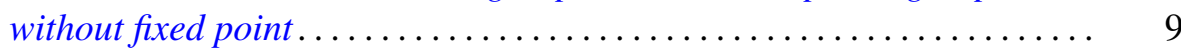

Homer Franklin Bechtell, Jr., Frattini subgroups and $\Phi$-central groups .... 15

Edward Kenneth Blum, A convergent gradient procedure in prehilbert

spaces ............................................

Edward Martin Bolger, The sum of two independent exponential-type random variables ...................................

David Wilson Bressler and A. P. Morse, Images of measurable sets .......

Dennison Robert Brown and J. G. LaTorre, A characterization of uniquely

divisible commutative semigroups........................

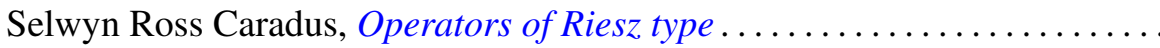

Jeffrey Davis and Isidore Isaac Hirschman, Jr., Toeplitz forms and ultraspherical polynomials ............................

Lorraine L. Foster, On the characteristic roots of the product of certain rational integral matrices of order two ......................

Alfred Gray and S. M. Shah, Asymptotic values of a holomorphic function



Sidney (Denny) L. Gulick, Commutativity and ideals in the biduals of

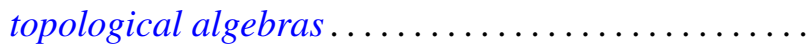

G. J. Kurowski, Further results in the theory of monodiffric functions

Lawrence S. Levy, Commutative rings whose homomorphic images are self-injective .

Calvin T. Long, On real numbers having normality of order $k$....

Bertram Mond, An inequality for operators in a Hilbert space. ...

John William Neuberger, The lack of self-adjointness in three-point boundary value problems ........................

C. A. Persinger, Subsets of $n$-books in $E^{3}$

Oscar S. Rothaus and John Griggs Thompson, A combinatorial problem in the symmetric group ............................... 175

Rodolfo DeSapio, Unknotting spheres via Smale .................. 179

James E. Shockley, On the functional equation

$$
F(m n) F((m, n))=F(m) F(n) f((m, n)) \ldots \ldots \ldots
$$

Kenneth Edward Whipple, Cauchy sequences in Moore spaces ... 